\title{
Das Subjekt und das Gegebene: Die Frage nach den Bedingungen der Möglichkeit der Erfahrung in der Transzendentalphilosophie und in der Phänomenologie
}

\author{
VITTORIO DE PALMA \\ Istituto Italiano per gli Studi Filosofici \\ $\triangle$ vittdepalma@hotmail.com
}

\begin{abstract}
[...] a mind which is one of the conditions of experience in general should be itself knowable and empirical. The alternative is transcendentalism, which explains knowledge by reference to a conditioning mind and [...] posits this mind as beyond or behind all experience. Such transcendentalism vitiates both epistemology and metaphysics. It vitiates epistemology because to suppose that the content of knowledge is informed or determined by some transcendent agent or principle, which is not to be found in experience, is to substitute a creation-myth for the analysis of knowledge. And it vitiates metaphysics by this invention of a transcendent agent which [...] it would be impossible to know. The ascription of the categories to a transcendent mind and of the matter of knowledge to transcendent things in themselves, are precisely similar fallacies. Those who hope to avoid the latter must, in all consistency, give up the former.

(C.I. Lewis, Mind and the World-Order.)
\end{abstract}

\begin{abstract}
In this article a comparison is made between the way the conditions of possibility of experience are conceived by Husserl and by Kantian and postKantian idealism. I show that - contrary to the latter - Husserl claims that the conditions of possibility of experience lie in the factually given sensuous contents, because sensuous syntheses, which are at the basis of the objectual
\end{abstract}


constitution, depend just on the peculiarity and the course of sensuous contents. Because of a conception of the relation between experience and thought that is antithetical to the idealistic one, Husserl holds that only experienceable (i.e., non-intellectual) forms can produce connections, which are not merely thought, but given in experience. What determines the structure of the world is therefore not the thinking or the understanding, but the sensuous given, which is independent from the subjective thought-activity and factual or contingent, since it cannot be traced back to a priori principles. The world-constitution is consequently an irrational fact, namely something that can be described, but not rationally explained.

Keywords Husserl, Kantian and post-Kantian idealism, conditions of possibility of experience, transcendental philosophy, facticity, constitution.

\section{Einleitung}

Der Aufsatz beabsichtigt aufzuzeigen, dass Husserls Auffassung der Bedingungen der Möglichkeit der Erfahrung der Auffassung von Kant und des deutschen Idealismus entgegengesetzt ist. Denn Husserl zufolge liegen solche Bedingungen im sinnlich Gegebenen: Die Struktur der sinnlichen Erfahrung beruht auf passiven Synthesen, die nicht vom Subjekt, sondern von der sachlichen Besonderheit und vom Verlauf der sinnlichen Erscheinungsinhalte abhängen. Hätte Erfahrung vor dem Eingreifen des Subjekts keine Struktur, könnte das Subjekt keine Welt konstituieren, weil Subjektakte keine sinnliche Form den sinnlichen Inhalten verleihen können und keinen sachlichen Zusammenhang zwischen den Erscheinungen herstellen können. Die apriorische Struktur der Erfahrungswelt wird also nicht durch den Verstand oder das Denken, sondern durch das faktisch Gegebene bestimmt, das nicht auf apriorische oder Vernunftgesetze zurückführbar ist. Demgemäß stellt die Weltkonstitution ein irrationales Faktum dar.

Der Aufsatz gliedert sich folgendermaßen. Nach einer Darstellung von Kants Gedanken des Verstandes als Grundes der Synthesis und Schöpfers der Natur (§ 2) wird der Idealismus in Anlehnung an Jacobis Ausführungen als Nihilismus charakterisiert, da er das sinnlich Gegebene in formale Bestimmungen auflöst, also das Reale im Denken vernichtet (§ 3). Es wird dann Hegels Kritik an Kants Auffassung der Dinge an sich sowie des Verhältnisses zwischen dem Logischen und dem Sinnlichen kurz vorgelegt und mit Husserls Ansatz verglichen: Genauso wie Hegel lehnt Husserl Kants Idee vom Ding an 
sich ab, aber im Gegensatz zu Hegel betrachtet er das Sinnliche als das Reale und schreibt dem Logischen keine reale Bedeutung zu (§ 4). Im nachfolgenden Abschnitt werden Kants und Husserls Konzeptionen der Formen und Gesetze der Erscheinungen gegenübergestellt: Im Gegensatz zu Kant meint Husserl, dass die Form der Erfahrung genauso wie der Inhalt erfahren wird und die Gesetze, unter denen die Erscheinungen stehen, in den Erscheinungen selbst liegen, da sie in der sachlichen Wesensbesonderheit der faktischen Erscheinungsinhalte gründen. Demzufolge stammen die weltkonstituierenden Zusammenhänge nicht aus dem Dativ, sondern aus dem Sachgehalt der Gegebenheiten (§ 5). Danach wird gezeigt, dass laut Husserl — anders als laut Kant — die Auflösung der Welt in ein Gewühl von Erscheinungen nicht nur durch subjektive Gründe, sondern auch durch objektive Gründe stattfinden kann. Die Weltkonstitution hat also nicht nur subjektive (d.h. in den Akten und Vermögen des Subjekts liegende), sondern auch objektive (d.h. in dem Inhalt der Erscheinungen liegende) Möglichkeitsbedingungen (§ 6). Es wird dann ausgeführt, dass Husserl zufolge das Prinzip der sinnlichen Konstitution nicht im Subjekt, sondern im sinnlich vorgegebenen Stoff liegt: Was sich konstituiert und was für eine Wesensstruktur es hat, hängt von der ichfremden Hyle ab, weshalb die Struktur der Welt nicht dem Subjekt, sondern dem faktischen Erscheinungsstoff entspringt (§ 7). Im Schlussabschnitt wird erläutert, dass die Rationalität (d.h. die apriorische Gesetzmäßigkeit, Begriffsmäßigkeit und somit Erkennbarkeit) der Welt kein Notwendiges, sondern ein Irrationales oder Faktisches ist, weil sie — anders als Fichte behauptet — auf die Bestimmtheit des sinnlich Gegebenen angewiesen ist, die nicht aus apriorischen oder Vernunftgesetzen erklärbar ist (§ 8).

\section{Der Verstand als Grund der Synthesis und Schöpfer der Natur}

Sellars bezeichnet den Mythos des Gegebenen als ,the idea that the categorial structure of the world [...] imposes itself on the mind as a seal on melted wax" (Sellars 1981, 12). Bei Kant sowie beim Großteil nachkantischen Denkens waltet der entgegengesetzte Mythos des gesetzgebenden Subjekts, wonach das Subjekt eine Struktur der Erscheinungswelt wie ein Siegel geschmolzenem Wachs einprägt. Diesem Mythos gemäß hat das sinnlich Gegebene in sich keine notwendige Struktur, die die Erfahrung und Erkenntnis des Subjekts bestimmt. Hierzu spricht Scheler von einer „Mythologie der erzeugenden Verstandestätigkeiten“, die auf der ,ganz mythologische[n] Annahme“ beruht, das Gegebene sei ein Chaos von Empfindungen, das erst mittels synthetischer Funktionen geformt werden müsste (Scheler 1966, 86, 72f.). 
Kant bemerkt, dass die Gesetzmäßigkeit der Erfahrung Voraussetzung der assoziativen Verknüpfung ist: Wäre die Folge der Erscheinungen keiner beständigen Regel unterworfen,

würde unsere empirische Einbildungskraft niemals etwas ihrem Vermögen Gemäßes zu tun bekommen, also, wie ein totes und uns selbst unbekanntes Vermögen im Inneren des Gemüts verborgen bleiben. Würde der Zinnober bald rot, bald schwarz, bald leicht, bald schwer sein, [...] so könnte meine empirische Einbildungskraft nicht einmal Gelegenheit bekommen, bei der Vorstellung der roten Farbe den schweren Zinnober in die Gedanken zu bekommen (Kant 1781/87, A 100f.).

Er löst jedoch die materialen Bedingungen der Erfahrung in die formalen auf, insofern er das „Ich denke“ (das die formale Bedingung der Synthesis darstellt) statt der sachlichen Struktur der Inhalte (die die materiale Bedingung der Synthesis darstellt) als Grund der Konstitution ansieht. Wie Stumpf (1891, 479f.) hervorhebt, behauptet Kant, die notwendige Bedingung aller möglichen Wahrnehmung sei, dass die Assoziation einen „objektiven Grund“ in der „Affinität“ der Erscheinungen hat, die ,an sich assoziabel“ sein müssen; er führt indessen diesen objektiven Grund auf die „Einheit der Apperzeption“, d.h. auf den „subjektiven Grund[] der Apperzeption“ zurück (Kant 1781/87, A 121f., 114). Er bezeichnet den Gegenstand als dasjenige, ,was da wider ist, dass unsere Erkenntnisse nicht aufs Geratewohl und beliebig, sondern a priori auf gewisse Weise bestimmt seien“; er fügt allerdings hinzu, dass ,die Einheit, welche der Gegenstand notwendig macht, nichts anders sein könnte, als die formale Einheit des Bewusstseins in der Synthesis des Mannigfaltigen der Vorstellungen“" (ebd. A 104f.).

Kants Ansatz beruht auf dem Vorurteil, dass Erfahrung eine bloß induktive Gesetzmäßigkeit aufweist (ebd. B 3f.) und lediglich ,den rohen Stoff sinnlicher Eindrücke“ liefert (ebd. A 1), die „,bloß subjektiv bleiben, und kein Erkenntnis des Objekts, mithin keine für jedermann gültige Vorstellung in der empirischen Anschauung darlegen“ (AA XX, 269): Erfahrung fordert „eine Synthesis der Wahrnehmungen, die selbst nicht in der Wahrnehmung enthalten ist“, denn in der Erfahrung kommen die Wahrnehmungen „nur zufälligerweise zueinander, so dass keine Notwendigkeit ihrer Verknüpfung aus den Wahrnehmungen selbst erhellt“" (Kant 1781/87, B 218f.). ${ }^{1}$ ${ }^{1}$ Selbst Hegel zufolge enthält die Erfahrung ,nur die Allgemeinheit einer solchen Er-
scheinung, nicht aber die Notwendigkeit des Zusammenhanges“, und ,lehrt also nur,
wie die Gegenstände beschaffen sind, nicht, wie sie sein müssen“ (HW IV, 209f.). 
Der Verstand schafft die Gegenstände und die Natur durch seine synthetischen Formen, da die Erscheinungen als solche keine Regel und kein Gesetz in sich haben (Stumpf 1891, 471). Denn sie ,stehen unter gar keinem Gesetze der Verknüpfung, als demjenigen, welches das verknüpfende Vermögen vorschreibt“" und Gesetze existieren nicht ,in den Erscheinungen, sondern nur relativ auf das Subjekt, dem die Erscheinungen inhärieren, sofern es Verstand hat" (Kant 1781/87, B 164). Obwohl nämlich das Mannigfaltige vor und unabhängig von der Synthesis des Verstandes gegeben ist (ebd. B 145), ist seine notwendige Verbindung „eine Verstandeshandlung“ (ebd. B 130) und „kann niemals durch Sinne in uns kommen“ (ebd. B 129): „Da die Zusammensetzung nicht in die Sinne fallen kann, sondern wir sie selbst machen müssen: so gehört sie nicht zur Rezeptivität der Sinnlichkeit, sondern zur Spontaneität des Verstandes“ (AA XX, 275f.). Demnach liegt Verbindung ,nicht in den Gegenständen, und kann von ihnen nicht etwa durch Wahrnehmung entlehnt und in den Verstand dadurch allererst aufgenommen werden" (Kant 1781/87, B 134f.). Die „Vorstellung der synthetischen Einheit des Mannigfaltigen [...] kann also nicht aus der Verbindung entstehen, sie macht vielmehr dadurch, dass sie zur Vorstellung des Mannigfaltigen hinzukommt, den Begriff der Verbindung allererst möglich“ (ebd. B 131). Diese Lehre gründet in der Annahme, dass Verbindung „nicht durch Objekte gegeben, sondern nur vom Subjekte selbst verrichtet werden kann, weil sie ein Actus seiner Selbsttätigkeit ist" (ebd. B 130). ${ }^{1}$ Insofern solche Handlung ,ursprünglich einig und für alle Verbindung gleichgeltend sein müsse" (ebd.), ist sie unabhängig von den Unterschieden in dem zu verbindenden Stoff, also von der Besonderheit desselben. ${ }^{2}$ Die Prinzipien der Synthesis des Mannigfaltigen liegen nämlich nicht in der

Demgemäß meint er, Assoziation sei willkürlich oder zufällig, also gesetzlos (HW X, $\S 455 \mathrm{~A})$.

${ }^{1}$ Kant bekräftigt wiederholt, dass ,im empirischen Begriffe des Zusammengesetzten die Zusammensetzung nicht vermittelst der bloßen Anschauung und deren Apprehension, sondern nur durch die selbsttätige Verbindung des Mannigfaltigen in der Anschauung gegeben und [...] vorgestellt werden kann“ (Brief an J. S. Beck, 17.X.1792, AA XI, 376). „,Das Zusammengesetzte nämlich kann, als ein solches, nicht angeschaut werden; sondern der Begriff oder das Bewusstsein des Zusammensetzens [...] muss vorgehen, um das mannigfaltige der Anschauung Gegebene sich in einem Bewusstsein verbunden, d.i. das Objekt sich als etwas Zusammengesetztes zu denken“ (Brief an J. H. Tieftrunk, 11.XII.1797, AA XII, 222f.). „Die Zusammensetzung können wir nicht als gegeben wahrnehmen, sondern wir müssen sie selbst machen: wir müssen zusammensetzen, wenn wir uns etwas als zusammengesetzt vorstellen sollen“" (Brief an J. S. Beck, 1.VII.1794, AA XI, 515).

${ }^{2}$ Vgl. Paton $(1936,258,505)$. 
Eigenart des Mannigfaltigen, sondern in der Natur des Verstandes, der seine eigenen Formen dem Mannigfaltigen aufnötigt. ${ }^{1}$

Kants Denken liegt die Vermutung zugrunde, dass „die Empfindungen ohne objektive Bestimmtheit sind“ (HW II, 310). ${ }^{2}$ Demgemäß meint er, die Form der Erfahrung sei unser „Zusatz“ (Kant 1781/87, B 1), weshalb „in der Erkenntnis a priori den Objekten nichts beigelegt werden kann, als was das denkende Subjekt aus sich selbst hernimmt" (ebd. B XXIII), mithin „wir von den Dingen nur das a priori erkennen, was wir selbst in sie legen“ (ebd. B XVIII). Es sind wir selbst, die die Gesetzmäßigkeit in die Erscheinungen hineinbringen (ebd. A 125). Denn „Erfahrung kann nicht gegeben, sondern muss gemacht werden" (AA XXI, 405; vgl. 322, 345, 366, 392, 394f.). Kant billigt nämlich das verum-factum-Prinzip, wonach ,wir nichts einsehen als was wir selbst machen können“"(AA XXII, 353). ${ }^{3}$ Die kopernikanische Wende besteht gerade in der These, dass ,die Vernunft nur das einsieht, was sie selbst nach ihrem Entwürfe hervorbringt", weshalb ,demjenigen, was die Vernunft selbst in die Natur hineingelegt, gemäß, dasjenige in ihr zu suchen“ ist, „was sie von dieser lernen muss“ (Kant 1781/87, B XIIIf.).

\section{Idealismus als Nihilismus}

Jacobi zufolge liegt der Kern der Kantischen Philosophie in der Einsicht, dass „wir einen Gegenstand nur insoweit begreifen, als wir ihn in Gedanken vor uns werden zu lassen, ihn im Verstände zu erschaffen vermögen“, weshalb nur Mathematik und Logik als Wissenschaften im eigentlichen Sinne gelten und „alle andern Erkenntnisse nur in dem Maße wissenschaftliche Eigenschaft erwerben, als sich ihre Gegenstände durch eine Art von Transsubstantiation in mathematische und logische Wesen verwandeln lassen" (JW III, 351). Insofern reine Vernunft ,ein Vernehmen [ist], das nur sich selbst vernimmt“, muss das Philosophieren der reinen Vernunft „ein chemischer Prozess sein, wodurch alles außer ihr in Nichts verwandelt wird, und sie allein übrig lässt“: „,der Mensch erkennt nur indem er begreift; und er begreift nur indem er — Sache

\footnotetext{
${ }^{1}$ Vgl. Paton (1936, 285f., 507).

${ }^{2}$ Eigentlich schreibt Kant den Empfindungen objektive Bestimmtheiten zu, die jedoch bloß empirisch sind und von den Dingen an sich abhängen: Alles Notwendige bzw. Apriorische in der Erfahrung ist auf das Subjekt zurückzuführen (Kant 1781/87, A 127f., B 69; Paton 1936, 139f.).

${ }^{3}$ Vgl. Reflexion 2394, AA XVI, 344; Reflexion 2398, AA XVI, 345; Brief an J. S. Beck, 1.VII.1794, AA XI, 515; Brief an J. Plücker, 26.I.1796, AA XII, 57. Zum verum-factum-Prinzip vgl. Löwith (1986).
} 
in bloße Gestalt verwandelnd - Gestalt zur Sache, Sache zu Nichts macht", denn um ein Wesen zu begreifen, „müssen wir es objektiv - als für sich bestehend - in Gedanken aufheben, vernichten, um es durchaus subjektiv, unser eigenes Geschöpf - ein bloßes Schema - werden zu lassen“ (ebd. 19-21). Demgemäß ist der Idealismus ein „Nihilismus“ (ebd. 44).

Abgesehen von der Weise, wie Jacobi sie entwickelt, und von seinen Absichten enthalten solche Ausführungen eine wertvolle Erkenntnis: Der Idealismus ist insofern ein Nihilismus, als er die realen bzw. vor dem Eingreifen des Subjekts gegebenen Sinnendinge in ideale bzw. durch die Denktätigkeit des Subjekts erzeugte Denkbestimmungen auflöst und vernichtet. Denn der Idealismus will ,im Idealen das Reale, in den logoi die onta begründen; so in Plato, so in Leibniz, so in Kant, der [...] den ganzen Begriff des Gegenstandes aus den Formalbestandteilen der Erkenntnis, aus dem Logischen im vertieftesten Sinne erst aufbaut" (Natorp 1973, 14). Der reale Gegenstand wird dadurch in formallogische Bestimmungen aufgelöst.

Kant ist insofern Idealist, als er leugnet, dass das Sinnliche das Wirkliche ist, und den Erscheinungen Dinge an sich zugrunde legt, die Abstraktionsprodukte, selbsterdachte Wesen oder Hirngespinste darstellen, und als er leugnet, dass das Sinnliche maßgebend ist, und die Gesetzmäßigkeit der Erfahrung auf die Subjektivität zurückführt, indem er behauptet, dass letztere ihre eigenen Formen den sinnlichen Inhalten verleiht. Der Idealismus hängt gerade mit der Degradation der Sinnlichkeit zusammen, die im nachkantischen Denken gipfelt. Denn letzteres erkennt dem sinnlich Gegebenen jede epistemische und ontologische Bedeutung $\mathrm{ab}$, da es irrational oder faktisch, nämlich aus Vernunftgesetzen unerklärbar ist. Fichte zufolge ist Sinnlichkeit „nur Versinnlichung [des Intelligiblen], nichts ursprüngliches“ (GA IV/2, 172) und „die Sinnenwelt ist ein Bild, das durchaus nichts weiter bedeutet, und gar keinen andern Zweck hat, als damit es zum einzigen wahren Bilde, das einen Gehalt hat, zum Bilde Gottes im Gesicht komme“ (GA I/10, 384). ${ }^{1}$ Er erklärt, dass der Idealismus ,eben Nihilismus sey, strenge Nachweisung des absoluten Nichts, außer dem Einen unsichtbaren Leben, Gott genannt" (GA II/17, 266f.). Hegel behauptet, dass „,nichts wirklich ist als die Idee“ (HW VII, 25) und „Gott nur das wahrhafte Sein ist“" (HW VIII, § 50 A), während die sinnlichen Dinge „,nur Schein“, also „nichts an sich sind“ (HW IX, 246 Z): Das Sinnliche ist „ein Nichtiges“ (HW V, 44) und gilt bloß als ,,anfangende Grundlage“, weil der sinnliche Stoff durch „,das Negative der Tätigkeit des Geistes [...] vergeistigt und als Sinnliches aufgehoben wird“" (HW X, § 442 A). Die Tätigkeiten des

\footnotetext{
${ }^{1}$ Die Fichte-Zitate werden nicht der neuen deutschen Rechtschreibung angepasst, sondern im Original angeführt.
} 
Geistes sind gerade „,verschiedene Weisen der Zurückführung der Äußerlichkeit zu der Innerlichkeit“, d.h. der „Idealisierung und Assimilation der Äußerlichkeit": Indem das Ich den ihm gegenüberstehenden mannigfaltigen Stoff erfasst, „wird derselbe von der Allgemeinheit des Ich zugleich vergiftet und verklärt, verliert sein vereinzeltes, selbständiges Bestehen und erhält ein geistiges Dasein“ (ebd. § 381 Z). Das Denken macht die äußeren Gegenstände dadurch ,zu einem Subjektiven, von uns Produzierten, uns Angehörigen“ (HW IX, § 246 Z), dass es sie in Denkgebilde verklärt und deren Selbständigkeit vernichtet. Die Erkenntnis besteht eben in der „Vernichtung der Äußerlichkeit“ (HW X, § 552 A), d.h. in der Auflösung des sinnlich Gegebenen in Denkbestimmungen.

Die Phänomenologie wälzt solchen Ansatz um. Gegen die Platonische, Christliche und rationalistische „Degradation der Sinnlichkeit“, wonach diese „ein Vermögen bloß verworrenen Vorstellens, die Quelle alles Scheins, Trugs, alles Irrtums und aller Schlechtigkeit" darstellt (Hua Mat III, 170), behauptet Husserl, Sinnlichkeit sei kein ,trübendes Medium, welches statt der Dinge an sich bloße Erscheinungen derselben gibt" (ebd. 172). Das Reale ist das Sinnliche (ebd. 168ff.) und ist also prinzipiell erfahrbar oder wahrnehmbar (Hua XVII, 457): Die Dinge sind nicht für uns, sondern an sich sinnlich, weshalb sinnliche Erfahrung „der Modus der Selbsthabe von Naturobjekten“ ist (ebd. 170) und „das in der Wahrnehmung wahrgenommene Ding das Ding selbst ist, in seinem selbsteigenen Dasein“ (ebd. 287). „Gegenstände sind als sie selbst in Erfahrung gegeben, Erfahrung ist Erfassung des Selbst" (Hua XXXIV, 308). „Die Dinge ihrem Sein und Wesen nach haben [...] keine Mysterien", denn obwohl sie im Lauf der Erfahrung immer neue Beschaffenheiten bieten, sind sie „etwas Gegebenes, etwas Wahrnehmbares, als das, was sie sind, Fassbares und nichts Verstecktes“, weil „,so geartetes Sein nur so zur Gegebenheit gebracht werden kann" (Hua XIII, 10). Das wahre Sein der Dinge besteht in ihrer Selbstgegebenheit, und nicht in einem Jenseits der möglichen Erfahrung (Hua XXXV, 276f.; Hua XXXVI, 67f.). Demzufolge stellt Wahrnehmung „,das letzte Maß der Wirklichkeit“ dar (Hua XL, 314) und ist „nichts zu Begründendes, sie ist dafür selbst Grund gebend“ (Hua XXIV, 8): „Nur Wahrnehmung hebt Wahrnehmung aus dem Sattel" (Hua XXXVI, 40). ${ }^{1}$

\footnotetext{
${ }^{1}$ Diametral entgegengesetzt ist der neukantianische Standpunkt: „Wer in der Wahrnehmung das natürliche Recht, die unmittelbare Gewissheit, die absolute Quelle der Wirklichkeit erblickt, der steht noch außerhalb der erkenntniskritischen Belehrung. Wenn Wahrnehmung das zureichende Kriterium der Objektivität enthielte, so würde es aller weiteren kritischen Zurüstung nicht bedürfen: wir wüssten alsdann, wo wir sie fassen könnten, die unendliche Natur" (Cohen 1984, 27).
} 


\section{Das Sinnliche, das Logische und das Ding an sich}

Wie Hegel bemerkt, meint Kant, dass die logischen Bestimmungen eine ,wesentliche subjektive Bestimmung" besitzen (HW V, 45), da die Wahrheit auf sinnlicher Realität beruht und erst die sinnliche Wahrnehmung den Gedanken Gehalt und Realität gibt (ebd. 38). Deswegen nimmt er - genauso wie der Empirismus - „die Erfahrung für den einzigen Boden der Erkenntnisse“ (HW VIII, § 40).

Puntel gibt folgendermaßen Hegels Grundargument gegen Kant wieder. Kants (meta)transzendentale Sätze beinhalten eine Präsupposition, die in ihnen selbst ausdrücklich negiert wird, denn sie nehmen den logischen Raum uneingeschränkt in Anspruch, andererseits aber schränken sie explizit ihn radikal ein. Das ergibt einen „Selbstwiderspruch“: ,indem gesagt wird, dass sich die Dimension der Dinge an sich unserer Erkenntnis entzieht, wird diese Dimension selbst angesprochen bzw. artikuliert" (Puntel 1983, 215). Bei Kant ist der logische Raum nicht mit dem Raum der Dinge oder des Wirklichen koextensiv, weil seine Auffassung der Sinnlichkeit als „restringierende Bedingung“ (Kant 1781/87, A 146/B 185f.) bewirkt, dass der logische Raum auf Funktionen des Subjekts eingeschränkt wird und der Bereich der Dinge nur die Rolle einer Materie der Erkenntnisse spielt. Daraus erfolgen „eine Aufspaltung des logischen Raumes in Formen des Subjekts, und zwar getrennt vom Inhalt" und „eine Aufspaltung des Bereiches der Dinge in eine Dimension des An-Sich und eine Dimension der Erscheinungen bzw. der Betrachtung der Dinge ,an sich selbst" und ,als Erscheinungen“" (Puntel 1983, 212).

Die Sinnlichkeit kann jedoch abgesehen von der Subjektivierung der Erkenntnis und der Ansetzung von Dingen an sich als restringierende Bedingung gelten: ${ }^{1}$ Weil die wirklichen Dinge an sich sinnlich sind, sind die logischen Formen keine ontologischen Formen, sondern bloß Formen der Dinge, sofern sie gedacht werden, d.h. Funktionen des Subjekts, die vom sinnlichen Gehalt getrennt sind und zu ihm hinzugetan werden. Formallogische Begriffe stellen ,eine der Sache äußerliche Reflexion“ dar (HW VI, 271) und sind „,nur subjektiv" (HW VIII, § 194 Z 1). Anders als Hegel meint, bleiben sie es und können sich nicht ohne äußeres Material objektivieren. Als ontologische Form der Dinge gilt nicht die logische Form, durch welche sie mittels der subjektiven Denktätigkeit umgestaltet werden, sondern die sinnliche Form, in der sie

${ }^{1}$ Da Kant eine transzendentale Untersuchung als eine Untersuchung von unserer menschlichen Erkenntnisart ansieht (Kant 1781/87, B 25; Martin 1969, 44), ist sein Denken durch den „Anthropologismus“ ausgezeichnet, wie Husserl hervorhebt.

9

Bull. anal. phén. XVII 6 (2021)

https://popups.uliege.be/1782-2041/ @ 2021 ULiège BAP 
unmittelbar, d.h. vor dem Eingreifen des Subjekts gegeben sind. Dadurch werden nicht die formale Unhintergehbarkeit und Universalität des Logischen, sondern nur dessen ontologische Tragweite geleugnet: Alles, was wir denken, denken wir im logischen Raum, also durch logische Formen, aber diese sind gerade bloß Formen des Denkens, nämlich von allem, sofern es gedacht wird. ${ }^{1}$ Denn wie Husserl bemerkt, gehören Denkbestimmungen nicht zu den Gegenständen, so wie sie in der Erfahrung gegeben sind, sondern nur so, wie sie durch logische oder Urteilsformen gedacht werden (Hua XVII, 405f.). Durch die logische oder Denkformung wird der sinnliche Gegenstand intellektiv gefasst und erhält eine nichtsinnliche Form, aber weder wird er in seinem eigenen Sein, d.h. in seinem sinnlichen Gehalt geändert noch wird ein neuer realer Gegenstand geschaffen (Hua XIX, 686f., 715f.). Das Logische hat demnach keine reale Bedeutung (ebd. 729; Hua XVII, 152). ${ }^{2}$

Im Gegensatz zum Transzendentalismus meint Husserl, dass Erfahrung eine vom Denken unabhängige Struktur hat und nicht gemacht werden kann, sondern gegeben werden muss. Daraus entspringt seine Idee einer transzendentalen Ästhetik, d.h. einer Beschreibung der sinnlichen Wesensform der Erfahrungswelt, die als die eigentliche Ontologie gilt. In der transzendentalen Ästhetik wird eine Beschränkung auf Körperwahrnehmung durch Ausschluss des urteilsmäßigen Wissens vollzogen (Hua XI, 295): Das Erfahren und das denkmäßige Bestimmen werden gegenübergestellt, um die Welt ,rein als erfahrene“, oder genauer die „Erfahrungsgestalt einer Welt überhaupt“ herauszufassen, „die eben notwendig überall voranliegt, zugrunde liegt, wenn über-

\footnotetext{
${ }^{1}$ Vgl. De Palma (2010).

2 Ähnlich bemerkt Lask, dass die logischen oder reflexiven Formen einen bloß ,enklitische[n], unselbständige[n], [...] parasitäre[n] Charakter“" haben (Lask 1923, 160), da sie das logisch nackte Material betreffen, verbrämen, umkleiden, umschließen und umgreifen, aber keineswegs durchdringen: Es „,bleibt, was es war, es ändert sich nicht seinem Gehalt und Wesen nach, es wird nicht in das verwandelt und verzaubert, wovon es lediglich umgeben und umgolten ist" (ebd. 75; vgl. 212f.). Logische Formen sind demnach ,,ohne sachliche Bedeutung'. Sie erreichen und treffen gar nicht die Wirklichkeit selbst“, denn sie sind durch die Subjektivität angestiftet und ,geben lediglich gewisse, erst in der Subjektivität erstehende Reflexionsbestimmungen, die von der Sache selbst nichts ahnen lassen“, weil die Inhalte nicht in ihrer spezifischen Eigenart, sondern ,lediglich als Elemente in der farblosen Region der bloßen Inhaltlichkeit überhaupt“ figurieren (ebd. 143f.). Ihr Material — das ,der bloße Schemen, das bloße Modell des ,Inhalts überhaupt”, des bloßen ,Etwas““ ist — stellt nämlich „,ein bloßes Geschöpf und Kunstprodukt der logischen Form selbst“" dar und hat „durch sie hindurch überhaupt erst Bestand“" (ebd. 140).
} 
haupt die Subjektivität dazu übergeht, über die von ihr erfahrene sich Gedanken zu machen, sie so oder so zu interpretieren“ (Hua XXXIX, 260), mithin um „das Ontische in seiner ont $<$ ischen $>$ Wesensart, wie es in der Erfahrung selbst beschlossen ist, herauszustellen“(Hua XLI, 346). Man muss demgemäß auseinanderhalten, „was Aktivität jeweils voraussetzt und was sie neu leistet“ (Hua XLII, 64), d.h. „was Sache der Vorgegebenheit und was Sache der Ichbeteiligung ist" (Ms. A VII 13/101b), um die subjektiven Zutaten, nämlich „das Moment des Begrifflichen“ (Hua XXIV, 319) abzuziehen und das vor dem Eingreifen des Subjekts Gegebene herauszupräparieren. Obwohl nämlich das Erfahrene nur durch Gedanken beschreibbar ist und ihm Niederschläge von Denktätigkeiten anhaften können, kann man immer zwischen dem sinnlich Erfahrenen einerseits und der daran geübten Denktätigkeit sowie den darin sich bildenden Gedanken andererseits unterscheiden (Hua IX, 57f.). Erfahren werden nicht die aktiv geschaffenen Denkgebilde, sondern nur das passiv Vorgegebene und in der Rezeptivität Erfassbare. „Z.B. ein prädikatives Gebilde ,Gold ist gelb“ ist nicht erfahren, aber das Gold ist eventuell erfahren und ebenso das Gelb. Nicht mehr: Gelb als Prädikat des Subjekts, Gold‘, die Subjektformung und Prädikatformung vollzieht das urteilende, beziehende Denken, in dem allein auch das Ist, der Ist-Verhalt, der ,Sachverhalt" entspringt" (ebd. 95f.; vgl. Hua XXIV, 318f.).

Was das Verhältnis zwischen Erscheinungen und Dingen an sich betrifft, behauptet Kant, dass der äußere Sinn ,nur das Verhältnis eines Gegenstandes auf das Subjekt in seiner Vorstellung enthalten könne, und nicht das Innere, was dem Objekte an sich zukommt" (Kant 1781/87, B 67). Was wir den erscheinenden Gegenständen beilegen, hängt nämlich „nur von der Anschauungsart des Subjekts in der Relation des gegebenen Gegenstandes zu ihm“ ab (ebd. B 69), weshalb ,die Dinge, die wir anschauen, nicht an sich sind, wofür wir sie anschauen, noch ihre Verhältnisse so an sich beschaffen sind, als sie uns erscheinen“: Alle Beschaffenheiten und Verhältnisse der Objekte in Raum und Zeit, ja selbst Raum und Zeit sind auf ,die subjektive Beschaffenheit der Sinne“" angewiesen und können ,als Erscheinungen nicht an sich selbst, sondern nur in uns existieren" (ebd. A 42/B 59). Husserl stülpt solchen Ansatz um, indem er den Raum als eine sachliche „Formbeschaffenheit aller Dinge“ betrachtet: „Er gehört zu ihnen und sie gehören zu ihm (als in ihm als der Form seiend), abgesehen von allen subjektiven Bedingungen der Anschauung“, denn es ist „keine subjektive Bedingung der Anschauung, dass Dinge nur gegeben sein können durch Anschauung" (Ms. B IV 1/33b). Die Unmöglichkeit, sinnliches Material unräumlich zu haben, besagt daher nicht „die Unfähigkeit, eine abweichende Anschauung zu bilden“, die in der Eigenart des Subjekts gründet, ,alles sinnliche Material in eine Raumform einordnen zu 
müssen“; sondern eine „wesensmäßige Unmöglichkeit“, die in der Eigenart des Dings gründet, weshalb ,nicht sinnliches Material notwendig räumlich geformt ist", sondern ,sinnliche Eigenschaften eines sinnlich gegebenen Dinges notwendig [...] räumlich sein müssen" (Hua VII, 357f.). Anders als Kant meint, ist also der Raum keine subjektive Form der Sinnlichkeit, mit der alle Erscheinungen insofern notwendig übereinstimmen, als sie nur durch dieselbe erscheinen und gegeben werden können (Kant 1781/87, A 93/B 125), mithin kein zwischen dem Subjekt und der Wirklichkeit vermittelnder Filter, sondern eine objektive Form der realen oder sinnlichen Gegenstände.

Was nur als Vorstellung in uns existiert, das sind nicht die Erscheinungen, die mit ihren sachlichen Beschaffenheiten ohne das Eingreifen des Subjekts gegeben sind, sondern die Dinge an sich, die subjektive Gebilde oder „leere Substruktionen eines [...] bodenlosen Denkens" darstellen und sich von den Gespenstern nur dadurch unterscheiden, dass sie „durch Erfahrung nicht gegeben, also auch durch sie nicht widerlegt werden können" (Hua XXXII, 216). ${ }^{1}$ Dinge, die nicht in der Erfahrung, sondern bloß im Denken bestehen, sind keine realen, sondern Gedankendinge. Kant spricht vom „Blendwerk“, die logische Möglichkeit des Begriffs der transzendentalen Möglichkeit der Dinge unterzuschieben (Kant 1781/87, A 244/B 302), und bemerkt: Ist der Gegenstand nicht gegeben, hat Erkenntnis keine „objektive Realität“", also ,sind die Begriffe leer, und man hat dadurch zwar gedacht, in der Tat aber durch dieses Denken nichts erkannt, sondern bloß mit Vorstellungen gespielt" (ebd. A 155/B 194f.). Wenn Kant Dinge an sich als Grund der Erscheinungen ansetzt, spielt er also mit Vorstellungen und vollzieht Blendwerke. Erfahrung ist der einzige Boden der Erkenntnisse.

\section{Formen und Gesetze der Erscheinungen}

Dem Transzendentalismus liegt die Annahme zugrunde, dass die ordnende Form der Empfindungen ,nicht selbst wiederum Empfindung sein kann“(Kant

\footnotetext{
${ }^{1}$ Fichtes und Hegels Kritik an Kants Ding an sich trifft in dem Maße zu, als sie sachlich auf empiristischen Argumenten beruht: Das Ding an sich kommt nicht in der Erfahrung vor und hat ,keine Realität, außer diejenige, die es dadurch erhalten soll, dass nur aus ihm die Erfahrung sich erklären lasse“, weshalb es eine „,bloße Erdichtung“ oder „,völlige Chimäre“ ist (GA I/4, 190, 192f.); es ist „,nur das Produkt des Denkens“, und zwar „des zur reinen Abstraktion fortgegangenen Denkens“ (HW VIII, § 44 A).
} 
1781/87, A 20/B 34), weil sie einen Zusatz des Subjekts darstellt. ${ }^{1}$ Husserl zufolge ist demgegenüber die Form der Erfahrung genauso wie der Inhalt erfahren: Die Ordnungsform der sinnlich gegebenen Inhalte ist selbst wiederum sinnlich gegeben, sonst könnten sie keine Ordnungsform aufweisen. Die Gesetze, unter denen die Erscheinungen stehen, liegen in den Erscheinungen selbst vor und unabhängig vom Eingreifen des Subjekts, da sie objektiv gegründet sind. Insofern also die Besonderheit des sinnlichen Stoffs das Prinzip der Konstitution darstellt, stammt die apriorische Struktur der Welt nicht aus subjektiven Formen, sondern aus der sachlichen Gesetzmäßigkeit der faktisch gegebenen sinnlichen Wasgehalte.

Dies kann unter Rückgriff auf Maimons scharfsinnige Ausführungen erläutert werden. Maimon bezeichnet das Gegebene als das, dessen Grund ,nicht im Erkenntnisvermögen, sondern außer demselben anzutreffen ist", d.h. dessen Entstehungsart ,sich nicht nach allgemeinen Gesetzen des Erkenntnisvermögens aus demselben erklären lässt“" (Maimon 1794, 203): Die rote Farbe ist gegeben, weil das Erkenntnisvermögen sie ,nicht aus sich selbst, nach einer von ihm selbst vorgeschriebnen Art, hervorbringen kann, sondern es sich dabei bloß leidend verhält" (Maimon 1790, 13). Das Gegebene ist nämlich die Materie der Anschauung, die „durchs Leiden“ entsteht, während deren Form „durch Tätigkeit“ entsteht (ebd. 417, 203). Denn die Ordnungsformen des Mannigfaltigen sind ,nicht [...] in einem besonderen Gegenstande gegründet; sondern in unserm Erkenntnisvermögen in Beziehung auf alle sinnliche[n] Gegenstände ohne Unterschied“" (ebd. 13). Solche Formen sind also transzendental, denn sie gehören nur insofern zum Gegebenen, als sie zum Subjekt gehören, das als Dativ der Gegebenheit fungiert. Husserl meint demgegenüber, dass die sinnlichen Formen, die die Struktur der Erfahrung ausmachen, nicht der Tätigkeit des Subjekts, sondern der Besonderheit der sinnlichen Inhalte entstammen und im selben Sinne wie diese gegeben sind (Hua XIX, 715). Ihr Grund ist darum außer dem Erkenntnisvermögen anzutreffen und ihre Entstehungsart lässt sich nicht nach allgemeinen Gesetzen des Erkenntnisvermögens aus demselben erklären. Solche Formen sind also nicht transzendental, sondern sachlich, denn sie gehören zum Gegebenen vermöge dessen Natur. Weltkonstituierende Zusammenhänge gründen demnach nicht im Dativ, sondern im Sachgehalt der Gegebenheiten.

\footnotetext{
${ }^{1}$ Selbst nach Fichte liegt der „Erklärungsgrund“ der Erfahrung „außer aller Erfahrung“ (GA I/4, 186-208), aber im Gegensatz zu Kant meint er, dass die Formen der Sinnlichkeit und die Kategorien nicht bloß im Ich vorhanden sind, sondern aus dem Ich deduziert werden (ebd. 201; GA I/3, 208; GA I/2, 262, 411). Deshalb wird Fichte von Hegel gelobt (HW XX, 401).
} 
Apriorische Gesetze sind nach Husserl relations of ideas, da sie nicht vom Subjekt, sondern von den Vorstellungsinhalten abhängen, d.h. „,mit den ,Ideen“ notwendig gesetzt sind“ (Hua VII, 359): ,wo die Ideen gegeben, da auch die Relation, in ewig unveränderlicher Weise" (Hua XXIV, 341). Gegen Kant behauptet er, dass apriorische Gesetzte selbst in der Sinnlichkeit walten (Hua XXXVII, 220ff.), weil es Vernunftwahrheiten gibt, die auf sinnlichen Vorstellungen beruhen (Hua XXVIII, 403). Nicht nur das Denken, sondern auch die Erfahrung besitzt daher eine Wesensgesetzmäßigkeit (ebd. 243): Neben logischen oder formalen Wesensgesetzen, die die Vernunft nach ihren möglichen Aktionen zum Thema haben und für die analytischen Denkzusammenhänge gelten, bestehen ontologische oder materiale Wesensgesetze, die Sinnlichkeit zum Thema haben und für die in der Erfahrung erscheinende Realität gelten (Hua XXXVII, 225f.; Hua XXIV, 293, 332f.; Hua XLI, 319f.). „Beiderseits sind Theorien von kantischem Typus [...] widersinnig“ (Hua XXXVII, 226). Anders als Kant meint, ist nämlich das Prinzip der synthetischen Urteile a priori keineswegs ,das Subjektive in der Form der Sinnlichkeit“ (AA XX, 272, 269) und somit ein „nur bloß subjektiv“ Gültiges (AA VIII, 220). Als Ideenrelationen gründen solche Urteile in den jeweiligen sinnlichen Wesensgehalten. Das materiale Apriori, das der Erfahrungsstruktur zugrunde liegt, ist gerade insofern „kontingent“, als es auf die faktische Gegebenheit des zugehörigen sinnlichen Wesensgehaltes (Farbe, Ton, Ding usw.) angewiesen ist (Hua XVII, 32ff., 379ff.; Hua XLI, 101). ${ }^{1}$

Entgegen der Transzendentalphilosophie - deren Grundgedanke ist, dass die Beziehungen zwischen den Phänomenen nicht gegeben sind, sondern vom Subjekt hergestellt werden $-^{2}$ unterscheidet Husserl zwischen logischen oder formalen Relationen, die von der sachlichen Eigenart der Fundamente unabhängig sind und durch Denktätigkeit geschaffen werden, und sinnlichen oder sachlichen Relationen, die in der Natur gegebener Inhalte gründen und vor jedem Verbindungsakt gegeben sind (Hua XIX, 288ff., 665ff., 714ff.;

\footnotetext{
${ }^{1} \mathrm{Zu}$ Husserls Auffassung des Apriori vgl. De Palma (2014). Husserl zufolge ist Hume mit seinen Ideenrelationen dem Begriff des Apriori nahegekommen und hat mit seinem Prinzip der Rückführung aller Erkenntnis auf Impressionen den Weg aller Ursprungsforschung angedeutet (Hua XXXVII, 224). Die Phänomenologie ist ein Versuch, aus diesen zwei Grundsätzen Humes einen eidetischen, also nicht naturalistischen Empirismus auszuarbeiten (De Palma 2021). In Husserls Denken liegt allerdings ein idealistisch-subjektivistisches Motiv, das mit dem in vorliegender Arbeit hervorgehobenen realistisch-objektivistischen Motiv in Konflikt steht. Zu diesem Konflikt vgl. De Palma (2016b); De Palma (2021).

${ }^{2}$ Vgl. Martin (1969, 115ff.).
} 
Husserl 1972, 214ff.). Im ersten Fall geht es um „ein subjektives In-Beziehung-Setzen und Bilden einer Beziehung“, im zweiten um eine „reale ,Beziehung “" (Hua XLI, 262), die eine materiale oder reale Einheit ergibt (Hua XIX, 291; Hua XXXI, 105). Sinnliche Einheitsformen liegen „vor der Vernunftfunktion der Begrifflichkeit und des Urteils“" (Hua VII, 224), da sie nicht durch das Denken gestiftet werden, sondern der sachlichen Besonderheit der sinnlichen Inhalte entstammen und in eins mit diesen gegeben sind (Hua XXXI, 101; Husserl 1972, 223, 296f.).

Indem Kant sich sträubt, das sinnlich Gegebene maßgebend werden zu lassen und die ausschlaggebenden Gründe der Synthesen im Erscheinungsstoff zu suchen (Stumpf 1891, 479), übersieht er, ,,dass viele inhaltliche Verbindungen uns gegeben sind, bei denen von einer synthetischen, die inhaltliche Verbundenheit schaffenden Tätigkeit nichts zu merken ist" (Hua XII, 41). Sinnliche Relationen - zu denen Verschmelzungen, Gleichheits- bzw. Ähnlichkeitsverhältnisse und alle sachhaltigen Wesensgesetze gehören - konstituieren sich nach denselben sachlichen Strukturgesetzen wie sinnliche Inhalte und sind ebenso passiv, d.h. ohne Eingreifen des Subjekts gegeben: „Unsere Geistestätigkeit macht nicht die Relationen; sie sind einfach da und werden bei gehöriger Richtung des Interesses bemerkt so gut als irgendwelche andere Inhalte“ (ebd. 42). Demnach „drängen sich die wahrnehmbaren Verhältnisse ebenso auf wie die absoluten Inhalte, sie sind uns mit ihnen gegeben, $[\ldots]$ sozusagen in sie eingebettet. Nicht das Bewusstsein ,stiftet ' Beziehungen zwischen unseren Empfindungsinhalten, sondern sie sind dem Bewusstsein gegeben, es hat sie nur zu konstatieren" (Stumpf 1939/40, 222).

Zwar ist das Gegebene das Ergebnis von Synthesen. Aber es geht dabei um passive Synthesen, die nicht von synthetischer Tätigkeit, d.h. von einem Verbindungsakt (Hua XLI, 128), sondern von den sinnlich vorgegebenen Inhalten hergestellt werden. Assoziative Synthesen, die die Apperzeption bestimmen, beruhen gerade auf der sachlichen „Eigenart der Elemente“ (Hua XI, 165), da sie in der Ähnlichkeit gründen, die eine Ideenrelation, d.h. ein erst von den Inhalten abhängiger Zusammenhang ist (ebd. 185, 285, 399f.; Husserl 1972, 215). „Die sinnliche Ähnlichkeit [...] ist die Resonanz, die jedes einmal Konstituierte begründet“ (Hua XI, 406). Insofern passive Geschehnisse „von selbst laufen“ und nur in dem Sinne „subjektiv“ sind, dass sie „aktivierbar sind durch die bloße Richtung des Ich darauf" (Ms. D 12/80a), ist die passive Konstitution ein „sachliches Geschehen“ (Ms. M III 3 I 1 I, § 6). ${ }^{1}$

Da die Konstitution der Objekte und der Natur ,,in bloßer Erfahrung und vor den kategorialen Aktionen“", d.h. passiv stattfindet (Hua XVII, 297), beruht

${ }^{1}$ Vgl. Holenstein $(1972,212,216)$. 
sie nicht auf Denk- oder Verstandesformen. Da letztere in der Erfahrung nicht gegeben sind, sondern Zutaten unserer subjektiven Denktätigkeit darstellen (ebd. 398; Hua XIX, 687), bestimmen sie keineswegs die Erfahrung. Denn sie ergeben keine sinnliche (wahrnehmbare) Struktur, weil sie nicht auf die Erfahrung selbst wirken, d.h. darauf, was und wie erfahren wird. Was und wie erfahren wird, hängt nicht von Denkformen, sondern von dem gegenwärtig und vergangen Gegebenen ab. Die Bildung von Apperzeptionen stammt daraus. „Erfahrung entspringt aus Erfahrung” (Hua XV, 211), und nicht aus Denken. Das Denken kann keine erfahrbaren Zusammenhänge zwischen den Erscheinungen herstellen, insofern die Denkspontaneität ,nichts an den sinnlichen Unterlagen“ ändern und keineswegs „,den Inhalt des unendlichen Laufs bestimmen oder gar ein erst Ungeordnetes geordnet machen" kann (Ms. B I 4/4b). Verstandes- oder Denkformen verleihen dem sinnlichen Stoff keine sinnliche Form: Da sie nicht in der sachlichen Eigenart der sinnlichen Inhalte gründen, sind sie nicht mit diesen gegeben und erzeugen also keine sachliche, d.h. nicht bloß gedachte, sondern gegebene Einheit.

Weil sie nicht im Subjekt, sondern in den Erscheinungsinhalten gründen, sind die apriorischen Formen der Erscheinungen ebenso wie die Erscheinungen sinnlich gegeben und stellen - anders als Kant in $\S 28$ der Prolegomena zu einer jeden künftigen Metaphysik behauptet — „Eigenschaften an Erscheinungen als Erscheinungen“ dar. Die Struktur der Erfahrung ist demzufolge nicht auf die Subjektivität, sondern auf die Beschaffenheit und den Verlauf der Erfahrungsinhalte angewiesen.

\section{Die Bedingungen der Möglichkeit der Erfahrungswelt}

Der Umstand, dass die Erscheinungen eine Ordnung aufweisen und kein Chaos bilden, hängt nach Kant von dem Subjekt, nach Husserl von dem sachlichen Inhalt und von dem sachlichen Verlauf der faktisch gegebenen Erscheinungen selbst ab.

Kant schreibt:

es könnten wohl allenfalls die Erscheinungen so beschaffen sein, dass der Verstand sie den Bedingungen seiner Einheit gar nicht gemäß fände, und alles so in Verwirrung läge, dass z.B. in der Reihenfolge der Erscheinungen sich nichts darböte, was eine Regel der Synthesis an die Hand gäbe, und also dem Begriffe der Ursache und Wirkung entspräche, so dass dieser Begriff also ganz leer, nichtig und ohne Bedeutung wäre (Kant 1781/87, A 90/B 123). 
Die transzendentale Deduktion beweist, dass dies unmöglich ist, weil das Subjekt den Erscheinungen eine objektive Struktur verleiht. ${ }^{1}$ Die Regelmäßigkeit der Erscheinungen ist insofern notwendig, als sie am Verstand liegt. Denn ohne die synthetische Einheit, die die Kategorien den Erscheinungen aufnötigen, gäbe es keine Gegenstände, sondern „ein Gewühl von Erscheinungen“ (ebd. A 111), „ein blindes Spiel von Vorstellungen, d.i. weniger als ein[en] Traum“ (ebd. A 112).

Husserl lehnt die transzendentale Deduktion zugunsten der empirischen Folgerung der Kategorien $\mathrm{ab}^{2}$ und hält für möglich, dass Erfahrung ein Gewühl von Erscheinungen sei: Weil Erfahrung ihre Struktur nicht dem Subjekt, sondern den faktischen Erscheinungsinhalten verdankt, könnte sie keine objektive Struktur aufweisen, wenn die Erscheinungsinhalte verschieden wären oder anders verlaufen würden. Die für die Weltkonstitution erforderliche RegelmäBigkeit der Erfahrung ist insofern kontingent, als sie nicht vom Subjekt hergestellt wird. Da also sie nicht im Subjekt, sondern „im Wesen der Erfahrung“ selbst liegen (Hua XVI, 141), sind die Bedingungen der Möglichkeit der Erfahrung erfahrbar, eidetisch und sachlich. Sie bestehen nämlich aus den „ontisch-apriorische[n] Wesensstrukturen, ohne die eine Welt als Welt möglicher Erfahrung undenkbar wäre" und die durch die „Methode der Wesensvariation“ zu gewinnen sind (Hua XXXII, 118). ${ }^{3}$ Die die Erfahrungsstruktur bestimmenden Kategorien - die Kant zufolge nicht in den Erfahrungsinhalten, sondern in dem sie erfahrenden Subjekt gründen, weshalb deren Natur, Zahl und Ursprung nur durch Rekurs auf Gott erklärt werden können $-{ }^{4}$ sind nicht einem Prinzip zu entnehmen, sondern in der Erfahrung selbst zu finden (Hua V, 25), weil sie nichts als die sinnlich gegebenen Wesensgehalte sind und somit objektiv begründet sind.

\footnotetext{
${ }^{1}$ Vgl. Paton (1936, 324 Anm. 3).

2 Vgl. Hua VII, 97ff.; Hua XXXV, 289; Hua XXXVII, 212; De Palma (2016a). Auch Lask meint in ausdrücklichem Gegensatz zu Hegel, das Differenzierungsprinzip der Seinskategorien sei „ein rein ,empiristisches““, nämlich die „Besonderheit des Materials“ (Lask 1923, 63, 59). In den Fortschritten deutet Kant selbst die empirische Folgerung an, indem er schreibt, dass die synthetische Einheit der Apperzeption oder des Bewusstseins, „nach Verschiedenheit der anschaulichen Vorstellungen der Gegenstände im Raum und Zeit, verschiedene Funktionen, sie zu verbinden erfordert, welche Kategorien heißen“" (AA XX, 276).

${ }^{3}$ Vgl. Hua XVII, 456; Hua VII, 385. Husserl versteht manchmal den Begriff ,transzendental“ eben als Synonym von "aus der Wesensintuition [...] geschöpft" (Hua XXXII, 123), d.h. von „eidetisch“".

${ }^{4}$ Vgl. Kant (1781/87, B 145f.); AA VIII, 249f.; Brief an M. Herz, 26.V.1789, AA XI, 52 .
} 
Gegen den Mythos des gesetzgebenden Subjekts schreibt Husserl: „erkennen wir begrifflich denkend und einsehend Gesetze, so sind wir nicht Gesetzgeber und die Dinge an sich ohne Gesetze, sondern der Welt selbst als seiender gehören die Gesetze zu, von ihr in ihrem Sein unabtrennbar" (Hua XXXII, 92). Um an die Welt apriorisch gültige Erkenntnisforderungen zu stellen, muss man aufweisen, dass solche Forderungen zunächst von der Erfahrungswelt selbst an die Erkenntnis gestellt werden (ebd. 247). Denn die Erfahrungswelt ist kein Inbegriff strukturloser Inhalte, der je nach dem angewandten Begriffschema eine verschiedene Form annimmt. Sie hat vielmehr ,gewisse intuitiv aufweisbare Strukturen [...], die uns binden, die an unser prädikatives Denken also Forderungen stellen“; deswegen ,,sind die an ein vernünftiges Erkennen zu stellenden Forderungen nicht solche, die wir leer formal deduzieren“, sondern ,solche, die die Welt selbst, die zunächst sinnliche, an uns stellt durch den ihr eigenen Sinn, sofern dieser ursprüngliche und erste Sinn (sinnliche Welt) in sich Möglichkeiten und Tendenzen zur Idealisierung als ,objektiv" in sich hat" (ebd. 101f.).

Wissenschaftliche Erkenntnis setzt voraus, dass die sinnliche Welt „tragfähig für das wissenschaftliche Denken“ und „logifizierbar“ ist, mithin eine „Antizipation“ einer exakten Welt darstellt (ebd. 97, 100f.): Hätte Erfahrung vor der Denkformung keine sachliche Gesetzlichkeit und wäre keine einheitliche, in sich zusammenhängende und standhaltende Welt schon durch sinnliche Erfahrung anschaulich gegeben, wäre keine Erkenntnis der Welt möglich, da sie keinen Boden für ihre höheren Objektivationen hätte (ebd. 15, 116; Hua XLI, 289; Hua IX, 56). Erfahrung kann nur dann in logisch-begrifflicher Weise erfasst werden, wenn vor und unabhängig von dem Eingreifen des Subjekts eine „Rationalität“ in den sinnlichen Gegebenheiten liegt (Hua Mat IX, 439). Denn die Rationalität oder Gesetzmäßigkeit kann nicht in die Erscheinungen hineingelegt werden, sondern muss ihnen immanent einwohnen, d.h. in eins mit ihnen gegeben werden. Bestünden keine standhaltenden Dinge, wären nämlich Logik und Mathematik nicht auf Erfahrung anwendbar (Ms. B IV 1/98b; Hua VII, 394f.). Zwar kann alles und jedes unabhängig von seiner sachlichen Besonderheit begrifflich bestimmt, verbunden, gezählt werden, aber das Bestimmen, Verbinden, Zählen setzten voraus, dass das Bestimmte, Verbundene, Gezählte als dasselbe identifizierbar ist, d.h. dass es nicht zerstäubt und eins bleibt - sie setzen insofern die Wirklichkeit des Seienden und der Welt voraus (Hua XXXVI, 23; Ms. A VII 20/43a). Erst das Bestehen materialer Wesenszusammenhänge ermöglicht demgemäß die Anwendung formaler Denkbestimmungen: Man kann der Erfahrung eine Denkform verleihen, nur weil sie vor und unabhängig von der Denktätigkeit eine sinnliche Struktur aufweist. 
Husserls Auffassung der Frage nach den Möglichkeitsbedingungen der Erfahrung ist demnach Kants Auffassung diametral entgegengesetzt. Sie lautet nicht: welche begrifflichen oder urteilsmäßigen Formen muss das Subjekt in die Erscheinungen hineinbringen, um eine objektive Welt herstellen und erfahren zu können? Sondern umgekehrt: „welche Formen muss Erfahrung selbst hinsichtlich der in ihr anschaulich erfahrenen Dingwelt haben, um begrifflich, urteilsmäßig, wissenschaftlich-wahrheitsmäßig erfassbar zu sein [...]?" (Hua XXXII, 97).

Wie Hume aufzeigt, ist die Gesetzmäßigkeit der Erfahrung eine Gesetzmäßigkeit der inhaltlich bestimmten Erwartung, da der in der vergangenen Erfahrung gegründete Vorgriff des Kommenden durch die gegenwärtige Erfahrung hindurchgeht und die Apperzeption bedingt. Die Induktion vom Nichtgegebenen aus dem Gegebenen gehört daher von vornherein zur Wahrnehmung, in der das wahrgenommene Objektfeld als „Abschlagszahlung“ für ein induziertes Zukunftsfeld fungiert (ebd. 144). Die Kraft der Erwartung und somit des Glaubens wird jedoch durch apriorische Gesetze bestimmt: Sie wächst zwar mit der Zahl der erfahrenen Fälle, also mit der Gewohnheit, aber dabei „ist gar keine Rede von dem Geiste des Menschen und von den Wirkungen, die er auf Grund der empirisch-psychologischen Gesetzmäßigkeiten erfährt" (Hua XXIV, 354), weil ,die auf Erfahrung sich gründenden Vermutungen unter Prinzipien stehen, die selbst den Charakter von Relationen zwischen Ideen haben" (ebd. 352). Finden wir, dass unter den gleichen Umständen stets gewisse ähnliche Tatsachen auftreten, dann konstituiert sich die allgemeine Erwartung, dass es immer so sei. Solche Erwartung kann zwar enttäuscht werden, aber ,solange die aktuelle Erfahrung ihren induktiven Stil bewahrt, [...] solange sind die allgemeinen Gewissheiten über unsere Willkür hinausliegende und uns durch ihre Rechtskraft eben rechtmäßig bestimmende" (Hua XXXII, 262). Demzufolge ist die ursprüngliche Form der Motivation, in der Ähnliches an Ähnliches erinnert und dessen Setzung motiviert, „eine ursprüngliche Vernunftform": Da Erwartung durch eine analogische Auffassung entsteht, die den Grund des propter auf ein post zurückführt, hat sie einen vernünftigen Grund in der vorangegangenen Erfahrung (Hua XIII, 356f.).

Würden die Dinge keine „Natur“ aufweisen und sich gesetzlos verändern, könnte ihre Identität weder gegenüber dem objektiven Wandeln noch gegenüber den wechselnden Erscheinungen durchgehalten werden (Ms. D 13 II/174a-b; Ms. B IV 1/95b). Dabei wäre die Konstitution einer seienden Welt unmöglich, wie das im Traum öfters der Fall ist, wo Dinge keine festen Bestimmungen und keine Identität besitzen. Die Weltkonstitution setzt voraus, dass die Reihenfolge der Erscheinungen einer Regel untersteht, nach der die künftigen Erscheinungen von den vergangenen vorgezeichnet werden, derart 
dass der weitere Erfahrungslauf von dem bisherigen induzierbar ist. Solche Regel kann jedoch nicht durch das Subjekt in die Erscheinungen hineingelegt werden.

Eine wirkliche Welt ist das Korrelat der einstimmigen bzw. einstimmig in infinitum fortlaufenden Erfahrung (Hua VIII, 457) und konstituiert sich zwar im Bewusstsein, aber entstammt ihm nicht. Die Einstimmigkeit der Erfahrung wird nämlich nicht vom Subjekt hergestellt, sondern ist in der Erfahrung selbst gegeben, da sie von dem faktischen Inhalt und dem faktischen Verlauf der Erscheinungen abhängt, die das Subjekt keineswegs bestimmen kann. Wären Inhalt und Verlauf der Erscheinungen, mithin das faktisch Gegebene verschieden, wäre die Welt verschieden oder würde sich keine wirkliche Welt konstituieren. Daraus stammt die Hypothese einer Weltvernichtung, d.h. einer Auflösung der Welt in ein „Gewühl von Erscheinungen“ durch Entfallen des regelmäßigen Stils der Erfahrung, der die Antizipation des Kommenden und damit die Gegenstandsapperzeptionen ermöglicht (ebd. 48f., 67f., 406, 453; Hua XVI, 288f.). Wenn nämlich der faktische Bewusstseinslauf ,die Identität durchzuhalten nie gestattet“, kann in seinem Zusammenhang keine „Transzendenz“ oder ,an sich seiende Realität" sich konstituieren, sondern bloß „eine ungefähre Dingeinheit“ (Ms. B IV 1/76a-b) und somit „,eine ,ungefähre“ Welt, die freilich streng nicht durchzuhalten ist" (Ms. D 13 II/174b). Es geht dabei um „rohe Einheitsbildungen“, d.h. um „vorübergehende Haltepunkte für die Anschauungen“, die unfähig sind, ,konservative ,Realitäten“, Dauereinheiten, die ,an sich existieren, ob sie wahrgenommen sind oder nicht", zu konstituieren" (Hua III, 103f.). Die Hypothese der Weltvernichtung beruht eben darauf, dass die Weltkonstitution aus objektiven Gründen unmöglich sein könnte:

Es ist denkbar, dass es im Erfahren von unausgleichbaren und nicht nur für uns, sondern an sich unausgleichbaren Widerstreiten wimmelt, dass die Erfahrung mit einem Male sich gegen die Zumutung, ihre Dingsetzungen einstimmig durchzuhalten, widerspenstig zeigt, dass ihr Zusammenhang die festen Regelordnungen der Abschattungen, Auffassungen, Erscheinungen einbüßt - dass es keine Welt mehr gibt (ebd. 103).

Könnte nicht im Bewusstsein alles an Elementarten [...] auftreten, was dem Begriff Vernunft Sinn gibt [...], und doch der mannigfaltige Inhalt des Bewusstseins sich nicht streng rationalisieren lassen oder überhaupt nicht, also keine Natur und Naturwissenschaft? Was nützen die idealen Möglichkeiten, die zum Urteil, zur Evidenz gehören, und die Normen, die sie gewähren, wenn ein „sinnloses Gewühl“ da ist, das in sich keine Natur zu erkennen gestattet? (Ms. D 13 II/200b; vgl. Ms. B IV 1/97a-98a). 
Die Möglichkeit einer Auflösung der Welt in ein Chaos von Erscheinungen besagt demnach die Möglichkeit, dass das Bewusstsein das Vermögen hat, vernünftig zu erkennen, aber wegen an sich unausgleichbarer Widerstreiten sein faktischer Inhalt keine Erkenntnis gestattet. Dem phänomenologischen Konstitutionsbegriff liegt gerade eine Gegenüberstellung von Bewusstsein und Inhalt zugrunde.

Die Konstitution einer objektiven Welt ist auf ,die Urfakta der Hyle“, und zwar auf das „Faktum“ angewiesen, „,dass das Urmaterial gerade so verläuft in einer Einheitsform“ (Hua XV, 385). Denn keine subjektive Leistung kann sinnliche Zusammenhänge erzeugen und bewirken, dass eine sachlich zusammenhangslose Folge von Erscheinungen gegenständlich erfahren wird. Durch seine Leistungen kann das Subjekt die Struktur der Erfahrungswelt nicht herstellen, sondern bloß erfassen und mittels kategorialer Formen denken. Welt und Gegenstände sind zwar das Korrelat subjektiver Akte. Damit aber das Bewusstsein Dinge in seinen Akten fassen kann, müssen feste sachliche Zusammenhänge zwischen den faktisch gegebenen Inhalten bestehen. Dass sich in einer Mannigfaltigkeit von Erscheinungen ein An-sich-Seiendes konstituieren kann, hängt nämlich von Inhalt und Lauf der Erscheinungen ab: Sind sie so beschaffen, dass sie ein sinnloses Gewühl ergeben, dann kann das Bewusstsein, wie immer es beschaffen ist, keine wirkliche Welt konstituieren.

Das ,,transzendentale[]“ Problem“ betrifft gerade die Weise, ,wie eine Welt als Natur sein kann ,an sich', wie ,bloße Dinge' in einer Natur im Voraus bestimmt sein können durch Erfahrung und Denken, wie im Voraus naturale Wahrheiten an sich gelten können" (Hua XLI, 301). Es geht dabei um die Weise, wie die Erscheinungen und ihr Lauf beschaffen sein sollen, damit sie das Ansetzen transzendenter Dinge und die Konstitution einer an sich seienden Welt gestatten. Die Frage nach den Bedingungen der Möglichkeit der Erfahrung ist demgemäß zunächst eine Frage nach dem faktischen Inhalt und dem faktischen Lauf der Erscheinungen, die keineswegs auf subjektive Bewusstseinsleistungen zurückführbar sind. „Dass diese Wahrnehmung da mit diesem Inhalt ist und dass sie so und so in andere Wahrnehmungen übergeht, das ist das brutum factum" (Ms. D 13 II/206b).

Die Konstitution einer wirklichen Welt hat nicht nur subjektive, sondern auch objektive Möglichkeitsbedingungen, da sie nicht nur voraussetzt, dass das Subjekt das Vermögen hat, Apperzeptionsakte zu vollziehen, sondern auch, dass der vorgegebene Stoff es zulässt. Deshalb sind in der Naturkonstitution die Seite der erkennenden Wesen und die der Natur selbst auseinanderzuhalten (Hua XXX, 309) und neben einer „Ichverrücktheit“ ist eine „Weltverrücktheit" anzusetzen (Hua XXXIX, 479). Die in $\S 32$ und 65-66 der Prolegomena zur reinen Logik in Bezug auf die logische Erkenntnis formulierte 
Unterscheidung zwischen noetischen, nämlich in den Akten gegründeten und objektiven, nämlich im Inhalt gegründeten Bedingungen der Möglichkeit ist demnach selbst auf die Weltkonstitution anzuwenden.

Die Relativität jedes Seienden auf die transzendentale Subjektivität ist also eine blo $\beta$ formale. Denn das transzendentale Ich „enthält keinerlei Prinzip, aus dem sich seine cogitata oder deren Wesensstrukturen ergeben könnten“, und fungiert als Geltungsgrund „nur in dem formalen Sinn, dass es der letzte Ort aller Ausweisung ist" (Tugendhat 1967, 212). Es ist daher nicht das Prinzip der Konstitution, sondern nur die letzte jeweilige Stätte aller Geltung und Ausweisung: Alles, was dem Ich rechtmäßig gelten soll, muss sich in den Akten des Ich geben und ausweisen, aber kann nicht aus dem Ich begründet werden (ebd. 199, 212ff.). Demgemäß sind die ontologische Ebene der Konstitution und die erkenntnismäßige Ebene der Ausweisung zu unterscheiden (ebd. 220ff.): Die Konstitution der Anderen für mich deckt sich nicht mit dem Sein der Anderen, die für sich oder absolut Seiende sind und sich in meinen Akten als unabhängig von meinen Akten konstituieren. Da also sich die Anderen für mich erkenntnismäßig in meinen Akten konstituieren, aber sie sich seinsmäßig nicht in meinen Akten konstituieren, fallen das „für mich“ und das „für sich“, d.h. die erkenntnismäßige und die ontologische Ebene nicht zusammen. Daraus ergibt sich, dass das ,Prinzip des erkenntnismäßigen Primats meines ego cogito universale Gültigkeit nur hat, wenn es nicht zugleich als ontologisches beansprucht wird“ (ebd. 222). Denn nur insofern ich das Sein für sich der Anderen nicht auf das Sein für mich der Anderen reduziere, kann ich die Anderen als transzendental ebenso wie ich ansehen, obschon sie nicht ,in Originalität und schlichter apodiktischer Evidenz, sondern in einer Evidenz äußerer Erfahrung gegeben sind“"(Hua I, 175), also kein Residuum der Reduktion bzw. kein Subjektives in erkenntnismäßigem Sinn bilden.

\section{Der vorgegebene Stoff als Prinzip der Konstitution}

Zur These der Absolutheit des Bewusstseins und der Relativität der Welt kommt Husserl durch die Variationsmethode. Denn die Möglichkeit eines Bewusstseins ohne Welt wird durch Abwandlung vom Inhalt des Bewusstseins gewonnen: Das von mir untrennbare Niveau der Erscheinungen könnte ,vielfältig gewandelt gedacht werden [...], ohne dass ich aufhörte zu sein, und darunter so, dass dieses Ich [...] erkennen würde: Das erscheinende Ding, die erscheinende Natur ist nicht, [...] es ist kein Rechtsgrund mehr, an einer Natur festzuhalten etc." (Hua XIV, 246). Dass Bewusstsein existieren kann, ohne dass eine transzendente Realität ist, ,sagt [...] nur: Ein einzelnes monadisches 
Sein ist denkbar ohne konstituierte Natur. Und ,denkbar' sagt: Wir können die immanente Zeit willkürlich besetzen, so dass keine Natur konstituiert wäre“ (Hua XXXVI, 79). Insofern also die zeitliche Synthesis ohne apperzeptive Synthesis bestehen kann, kann das Bewusstsein als einheitlicher Erlebnisstrom bestehen, auch wenn die die Zeitform erfüllenden Inhalte sachlich zusammenhangslos sind und ihr Lauf keine Ding- und Weltkonstitution ermöglicht. Bestünde zwischen den Erscheinungsinhalten keine sachliche Verbundenheit, dann könnten im Bewusstsein kein objektiver Sinn und keine Erkenntnisbildung entspringen. Die Konstitution einer seienden Welt ist demgemäß auf die inhaltliche Besetzung der Zeitform angewiesen, die keineswegs durch das Bewusstsein bestimmt werden kann. „Die Hyle kommt zufällig, sie steht nur unter den allgemeinsten Wesensgesetzen kontinuierlicher Zeiterfüllung" (Hua XIV, 14). Dass Bewusstsein ohne Welt bestehen kann, heißt also bloß, dass formal-zeitliche Synthesen (die unabhängig vom Inhalt sind) ohne sachlichassoziative Synthesen (die vom Inhalt bedingt werden) bestehen können. In diesem Fall gäbe es eine bewusste Sukzession von Erscheinungen, aber die Weltkonstitution wäre unmöglich, weil sich eine ,einstimmig setzbare, also seiende Welt" (Hua III, 497) durch sachliche Synthesen konstituiert, die nicht im Bewusstsein, sondern im Inhalt gründen. Das Bewusstsein mit seinen Leistungen ist daher notwendige, aber nicht hinreichende Bedingung der Konstitution einer objektiven Welt.

Die Unterscheidung zwischen formalen und sachlichen Synthesen liegt auch der Ansetzung einer Auflösung des personalen Ich zugrunde. Denn „Ich als $I c h$ habe Einheit durch die Welt, wenn sie wirkliche Welt ist, wenn sie Titel für ein Reich der Wahrheiten an sich ist" (Ms. A VI 30/38b), weil Bedingung der Konstitution meines personalen Ich ist, dass eine einstimmig bestimmbare „Objektwelt für mich beständig erhalten bleibt“ (ebd. 54b), d.h. eine „Natur ,an sich““ besteht (ebd. 49a). „Ohne Objekt bin ich nicht Ich“(ebd. 54a). Wenn nämlich die Dinge und ihre Bestimmungen gesetzlos wechselten, könnten für mich wahre Objekte nicht bestehen und ich wäre kein identisches Subjekt meiner Akte, sondern „,ein ,vielfärbiges“ Selbst“", d.h. ein Ich-Pol, der ,keinen personalen habituellen Sinn“ hat, obwohl ich die formale „Einheit meines Lebens, die Mannigfaltigkeit meiner Empfindungsdaten in der Einheit der immanenten Zeit" hätte: „Ich bin, aber ich bin ich nicht in Bezug auf eine Natur, eine Welt und nicht in einer Welt, weltlos", weshalb mein Leben mitsamt der Ich-Polarisierung verbleibt, wenngleich es ein „objektiv sinnloses“ ist (ebd. 52b).

Zeitliche Daten haben eine ,außerzeitliche Materie“ sowie ,außerzeitliche Bestimmungen“ (Hua X, 63, 65), die „das bewusstseinsfremd Gewordene, Empfangene" darstellen und keineswegs vom Bewusstsein geschaffen werden (ebd. 100). Ohne zeitliche Synthesis kann kein Inhalt gegeben sein. Was aber 
die sachliche Struktur der Erfahrungswelt bestimmt, d.h. was bewirkt, dass etwa ein Rotmoment oder ein Blaumoment erscheint, liegt nicht an der zeitlichen Synthesis, die als bloß formal-universale Synthesis alle Inhalte unabhängig von ihrer sachlichen Besonderheit verbindet. Zeitbewusstsein ist nämlich „nur ein eine allgemeine Form herstellendes Bewusstsein“ (Husserl 1972, 76, 207), weil die Zeitform - genauso wie das leere Etwas der formalen Ontologie - eine allgemeinste Form jedes erdenklichen individuellen Inhalts ist, der je nach seiner sachhaltigen Eigenart verschieden ist (Hua XI, 138, 333f.; Hua Mat VIII, 295ff.). ${ }^{1}$ Deshalb ,ist die intentionale Analyse des Zeitbewusstseins und seiner Leistung von vornherein eine abstraktive“: Da sie „von dem Inhaltlichen abstrahiert“, kann sie nicht sagen, ,was dem jeweiligen Gegenstand inhaltliche Einheit gibt, was Unterschiede des einen und anderen inhaltlich ausmacht" (Hua XI, 128), d.h. was die Apperzeption bestimmt.

Was sich konstituiert, hängt von der sinnlich vorgegebenen ichfremden Hyle $a b$, die den ,irrationalen Gehalt des Bewusstseins“ bildet (Hua XIV, 291): Da der Inhalt des Seins und der Seinsformen durch den irrationalen Stoff bestimmt wird (Hua XXVIII, 226; Hua Mat IX, 196f.), ist die Konstitution auf das „empirisch Zufällige“ oder „materiale Faktum“ angewiesen (Hua XIV, 306). Die Struktur der Hyle hängt demnach nicht vom Subjekt, sondern von der Beschaffenheit der Hyle ab. Solche Beschaffenheit, die die Konstitution bestimmt und durch das Subjekt nicht bestimmt wird, ist ein letztes und unerklärbares Faktum. „Ursinnlichkeit, Empfindung etc. erwächst nicht aus immanenten Gründen, aus seelischen Tendenzen, sie ist einfach da, tritt auf" (Hua IV, 335).

Die Hyle besteht im „Nicht-Ich“, das ein „Ungeistiges“ ist und ohne das kein Ich möglich ist (Hua XIII, 92; Hua XIV, 379, 244, 52). Denn „,notwendig gehört zum Ich ein Reich der Gegenständlichkeiten, die nicht selbst Bewusstsein oder gar Ich sind“: Das Objekt, d.h. ,das ichfremde Individuelle“ ist „bewusst, aber nicht Bewusstsein“ und somit nicht „ichlich, subjektiv“, obwohl es ,im Subjektiven des Bewusstseins Bewusstes“ ist (Hua XIV, 51 und Anm. 2). Da es nicht ,als reell einig mit dem Subjektiven sein kann“ und „es sich als etwas gibt, was vom Vermeintsein und Wahrgenommensein unabhängig ist (wenn es in Wahrheit ist)“, heißt das Transzendente ,im prägnanten Sinn objektiv, dem Ich und allem Subjektiven, allem untrennbar und reell mit dem Ich

\footnotetext{
${ }^{1}$ Solchen bloß formalen Charakter teilt die zeitliche Synthesis mit der kategorialen Synthesis: Genauso wie die kollektive Verbindung eine „lose und äußerliche“ ist gegenüber der inhaltlichen Verbindung (Hua XII, 20), ist die zeitliche Einheit eine „losere“ gegenüber der assoziativen Einheit (Hua Mat VIII, 297), die ,inhaltliche Bedingungen" hat (ebd. 9).
} 
und Ichleben Einigen, gegenüberseiend als nicht-subjektiv“ (ebd. 52). Das Bewusste ist also nicht selbst Bewusstsein oder Bewusstseinsimmanentes, denn „für mich ist, was ich selbst nicht bin, aber was ich in meinem Sein [...] bewusst habe als Nicht-Ich. [...] Das Subjektive ist Bewusstsein von Nichtsubjektivem, und die Zeitigung ist auch Zeitigung dieses Nichtsubjektiven“" (Hua Mat VIII, 361). Demgemäß bildet das Nicht-Ich den ,nichtsubjektive[n] Kern“ der Konstitution (ebd.) und das transzendentale Ich ist ,ein Relatives, eine ichliche Struktur gegenüber dem, was dem Ich vorgegeben ist" (ebd. 59), weil ,es zum Wesen des Ich gehört, auf ein ihm Fremdes (in einem eigenen Sinn, Äußeres") angewiesen zu sein und reizbar zu sein“ (Ms. E III 2/22a). Das „Ichfremde" oder ,Hyletische“ ist gerade das, was ,dem puren Ich vorgegeben“ ist (ebd.), es „affiziert“ und somit „,das Subjekt zu Tätigkeiten motiviert“ (ebd. 22b).

Jedes Erlebnis ist insofern „zweiseitig“, als es eine „ichliche“ und eine „,ichlose“, „,ichfremde“ oder „hyletische“ Seite hat, die die Seite des Inhalts darstellt (Hua Mat VIII, 183, 188f.), Als „,das letzte Ichfremde“ gelten „die ,sinnlichen Daten“ in ,sinnlichen Feldern““(ebd. 295), d.h. die „ständige Kernschicht der ,Sinnesfelder", des Vor-Ichlichen“, welche in aller ichlichen Leistung vorausgesetzt ist als ihre „letztliche [...] Materie“ (Hua XXXIX, 432). Das Korrelat des Aktbewusstseins ist gerade „ein nicht-ichliches, ein sachliches Wahrnehmungsfeld" (Hua XLII, 54), das den Aktvollzug bestimmt. Denn die ichfremde sinnliche „Materie“ fungiert als „Reiz zum Aktus“ (Hua XXXIX, 433) und somit als Quelle der Intention: Letztere wird vom vorgegebenen sinnlichen Inhalt bedingt, weil sie sich auf eine Abgehobenheit richtet, die eine Affektion ausübt und das Zuwenden des Ich motiviert (Hua XI, 84f.; Hua IX, 131, 209; Hua XXXVII, 332). Akte stellen demnach eine „Antwort“ auf eine Affektion dar, ${ }^{1}$ da sie durch Affektionen oder Impressionen, also durch das ,ichfremd Affizierende“ motiviert sind (Hua XI, 342; Hua IV, 336). Gegenstände werden durch Akte erfasst, Akte kommen jedoch durch affizierende Inhalte zustande, die das wahrhafte Erste darstellen: Ohne inhaltliche Abhebungen gibt es keine Affektionen und ohne Affektionen keine intentionalen Akte. Die Urquelle der Intention liegt darum in den passiv gegebenen Gliederungen des Wahrnehmungsfelds, also in den sachlichen Zusammenhängen zwischen sinnlichen Inhalten. ${ }^{2}$

Indem er die Faktizität der Konstitution erörtert, schreibt Husserl:

${ }^{1}$ Vgl. Hua XI, 166f.; Hua Mat VIII, 184, 189, 191; Hua XLII, 28, 34, 35.

${ }^{2}$ Vgl. De Palma (2015). 
Wir kommen auf letzte „Tatsachen“ — Urtatsachen, auf letzte Notwendigkeiten, die Urnotwendigkeiten. Aber ich denke sie, ich frage zurück und komme auf sie schließlich von der Welt her, die ich schon habe. [...] Ich bin das Urfaktum in diesem Gang, ich erkenne, dass zu meinem faktischen Vermögen der Wesensvariation etc. in meinem faktischen Rückfragen sich die und die mir eigenen Urbestände ergeben, als Urstrukturen meiner Faktizität. Und dass ich in mir einen Kern von „Urzufälligem“ trage in Wesensformen [...]. Mein faktisches Sein kann ich nicht überschreiten (Hua XV, 385f.).

Letzte oder Urtatsachen sind „mein urtümliches Ich“ und „mein urtümliches Nicht-Ich“, die als „Urvoraussetzungen“ der Konstitution gelten (Hua Mat VIII, 199). Deren Beschaffenheit ist zufällig und somit unerklärbar: Sie könnte verschieden sein und keine Weltkonstitution gestatten. Insofern also ihre Konstitution nicht notwendig, sondern kontingent ist, ist „die Welt ihrer Existenz und ihrem Sosein nach ein irrationales Faktum“ (Hua XVI, 289). Das Faktische ist eben das „Irrationale“ (Ms. B I 4/2b), d.h. das, was auf apriorische oder Vernunftgesetze nicht zurückführbar ist (Hua XXXVI, 18).

\section{Der irrationale Grund der Rationalität}

In der 1812 gehaltenen Vorlesung über die Transzendentale Logik schreibt Fichte:

Durch den Begriff, in jeder Rücksicht[,] ist ja die Welt gesetzt, und diesen muss sie ausdrücken, ausserdem wäre sie nicht: was aber durch den Begriff bestimmt ist, ist ja nicht das empirische, sondern für das empirische bleibt nur übrig dasjenige, was durchaus nicht durch den Begriff bestimmt ist. Es kommt in dieser Einsicht nur darauf an, dass man diese beiden Bestandtheile des faktischen Seyn[s] wohl scheide. [...] Das empirische ist seinem Daseyn nach zwar, durchaus aber nicht seiner Bestimmtheit nach, begreiflich. Diese Unbegreiflichkeit, mithin Absolutheit für den Verstand ist es nun, welche von jeher veranlasst hat, u. noch veranlasst[,] dieselbe für ein absolutes Seyn zu halten, und welche allen Dogmatismus [...] herbeigeführt hat (GA II/14, 334).

Das Empirische seiner Bestimmtheit nach sei jedoch „nur darum unbegreiflich [...], weil es das absolute Nichts ist; lediglich dazu da im Begriffe, um am Gegensatze mit demselben das wahrhaftige Was zu verstehen, als solches", denn erst „,die Begriffmäßigkeit [...] muss seyn [...]: u. ausserdem ist keine Welt möglich" (ebd. 334f.). Der faktische Inhalt ist irrelevant, insofern es keine Notwendigkeit in sich trägt: 
Wir haben das ganze faktische [...] in seiner Form eingesehen, als durchaus nothwendig [...]. Bloß mit dem Unterschiede, daß wir für den einen Theil des Faktum, die Ichheit, das bestimmte, u. qualitative Gesez in seinem Inhalte, einsehen; für den faktischen Inhalt dieser SichAnschauung des Ich, nur überhaupt einsehen, daß eine seyn müsse; dafür aber, daß gerade diese sey, kein Gesez haben: zugleich aber auch scharf einsehen, daß es ein solches Gesez gar nicht geben könne, daß darum das qualitative Gesez für diese Bestimmtheit eben die Gesezlosigkeit selbst sey. Wird nun das notwhendige als a priorisch genannt, so haben wir in diesem Sinne durchaus die ganze Fakticität als apriorisch eingesehen (ebd. 337f.).

Fichte bemerkt anderswo: „Es ist das Grundgebrechen aller, ihre Grenzen verkennenden, vermeintlichen Wissenschaft (des transzendentalen Verstandesgebrauchs), wenn sie sich nicht begnügen will, das Faktum rein als Faktum zu nehmen, sondern es metaphysiziert" (GA I/9, 188). Er selbst metaphysiziert jedoch das Faktum, indem er ein Zufälliges als ein Notwendiges hinstellt. Die Begriffsmäßigkeit, d.h. die apriorische Form der Erfahrungswelt ist nämlich keine Notwendigkeit, sondern ein Faktum, weil sie auf ,den faktischen Inhalt“ der Erscheinungen angewiesen ist. Die Erfahrung könnte ein Gewühl von Erscheinungen sein, das keine Begriffsmäßigkeit aufweist - genauso wie ein Traum, der neben Regelmäßigkeiten die sinnlosesten Aufeinanderfolgen bringt: „Während wir mit einem Bekannten sprechen, verwandelt er sich in einem anderen, zuweilen verwandeln wir uns sogar selbst, auch die Umgebung, die ganze Gegend nimmt im Handumdrehen neue und groteske Formen an usf." (Stumpf 1939/40, 601). Weder Naturgesetze noch Begriffe wie Substanz und Kausalität noch Prinzipien wie der Satz vom Widerspruch sind auf derartige Erscheinungen anwendbar, in denen sich kein Ding und keine Welt konstituieren können.

Das ,wahrhaftige Was" ist der sinnliche Sach- oder Wasgehalt des jeweilig Gegebenen, der ein Zufälliges oder Faktisches, also ein aus Begriffen und Prinzipien Unableitbares ist. Weit davon, „das absolute Nichts“ zu sein, wie Fichte nihilistisch behauptet, ist das empirisch Gegebene seiner empirischen Bestimmtheit nach dasjenige, das die Wesensstruktur der Erfahrungswelt bestimmt und deren Erkenntnis ermöglicht, insofern es die sachlichen $\mathrm{Zu}$ sammenhänge zwischen den Erscheinungen ergibt. Die Begriffsmäßigkeit der Welt ist auf die zufällige Bestimmtheit des Gegebenen angewiesen. Der Grund der Rationalität oder apriorischen Gesetzmäßigkeit der Welt ist demzufolge ein Irrationales, d.h. etwas, das anders sein könnte und nicht aus apriorischen oder Vernunftgesetzen erklärbar ist. Es geht um ein Faktum, das rein als Faktum zu nehmen, also bloß festzustellen ist. Jeder Versuch, es wegzudeuten, ist eine idealistische Täuschung. 
Als „Norm der Möglichkeit“ ist das Apriori „,vom Grund aus gestaltend für die Methode der Rationalisierung des Faktums“, die „lehrt, das Faktum rational zu ,erklären', nämlich es aus Prinzipien verständlich zu machen“" (Hua XLI, 320). Nun, in einem späten Text bezeichnet Husserl ,[d]as Faktum, das Irrationale als Voraussetzung aller Rationalität" (Hua XLII, 66) sowie als das „letztlich Wirkliche[]“ (ebd. 67) und in Bezug darauf, dass die Konstitution in der passiven Einheitsbildung gründet, schreibt er: „Irrationales Faktum“ alles letztlich faktisch, also auch die Wesensstruktur, als Prinzip der Erklärungen“ (ebd.).

Als ichfremd kann das sinnlich Gegebene bzw. das Wirkliche durch Denktätigkeit erfasst, aber nicht geschöpft oder verändert werden. Für es gilt das, was Hegel über die Natur als das Andere der Idee sagt: Es zeigt „keine Freiheit, sondern Notwendigkeit und Zufälligkeit" (HW IX, § 248). ${ }^{1}$ Deshalb kann es in seiner sachlichen Beschaffenheit und sachlichen Wesensstruktur beschrieben oder aufgeklärt, aber nicht erklärt werden. Es gilt also nicht, schimärische Erklärungen zu ersinnen, die das Gegebene und seine apriorischen Formen im Subjekt begründen. Es gilt vielmehr, sachliche Gegebenheiten festzustellen, wobei mit „Gegebenheiten“ nicht nur einzelne Inhalte bezeichnet werden, sondern auch Strukturzusammenhänge, die zwischen den einzelnen Inhalten bestehen, in der Natur letzterer gründen und ebenso wie letztere sinnlich erfahren werden.

Danksagung Ich danke der Direktorin des Husserl-Archivs in Löwen, Prof. Julia Jansen, für die Genehmigung, aus Husserls unveröffentlichten Manuskripten zu zitieren, sowie Wolfgang Kaltenbacher für die Hinweise zur Verbesserung des Textes.

\footnotetext{
${ }^{1}$ Die Ohnmacht des Begriffs (d.h. des Denkens), die Zufälligkeiten und Besonderheiten der Natur (d.h. der Wirklichkeit) zu begreifen, konstruieren und deduzieren, gibt Hegel mittels eines idealistischen Blendwerks für die „Ohnmacht der Natur“ aus, ,den Begriff in seiner Ausführung festzuhalten“ (HW IX, § 250 A; vgl. HW VI, 282). Dadurch gibt er implizit zu, dass die Welt ,nicht aus bloßen Kategorien oder reinen Begriffen, [...] sondern aus konkreten und zufälligen Dingen“ besteht, weshalb es ,um das Unlogische“ geht, nämlich „um jenes andere, das nicht der Begriff ist, sondern sein Gegenteil, welches den Begriff gleichsam unwillig annimmt“" (Schelling 1972, 225).
} 


\section{Abkürzungen}

AA Kants Gesammelte Schriften. Hg. von der Preussischen Akademie der Wissenschaften. Berlin: Reimer (dann De Gruyter), $1900 \mathrm{ff}$.

GA Fichte, J. G., Gesamtausgabe der Bayerischen Akademie der Wissenschaften. Stuttgart-Bad Cannstatt: Fromman-Holzboog, 19622012.

Hua Husserliana. Edmund Husserl - Gesammelte Werke. Den Haag: Nijhoff (dann Kluwer, dann Springer), 1950 ff.

Hua Mat Husserliana Materialien. Edmund Husserl - Materialien. Dordrecht: Kluwer (dann Springer), $2001 \mathrm{ff}$.

HW Hegel, G. W. F., Werke in zwanzig Bänden. Hg. von E. Moldenhauer und K. M. Michel. Frankfurt: Suhrkamp, 1969-1971.

JW Jacobi, F. H., Werke. Hg. von F. Köppen und F. Roth. 6 Bde. Leipzig: Fleischer, 1812-1825.

\section{Literatur}

Cohen, H. (1984). Das Prinzip der Infinitesimal-Methode und seine Geschichte. Ein Kapitel zur Grundlegung der Erkenntniskritik. In Werke. Bd. V/1 (1-162). Hildesheim/Zürich/New York: Olms.

De Palma, V. (2010). Die Kategorien des Sinnlichen. Zu Husserls Kategorienlehre. Phänomenologische Forschungen: 23-39.

De Palma, V. (2014). Die Fakta leiten alle Eidetik. Zu Husserls Begriff des materialen Apriori. Husserl Studies 30: 195-223.

De Palma, V. (2015). Der Ursprung des Akts. Husserls Begriff der genetischen Phänomenologie und die Frage nach der Weltkonstitution. Husserl Studies 31: 189212.

De Palma, V. (2016a). Subjekt und Erfahrung. Grundlagen und Implikationen von Husserls Kritik an die transzendentale Methode Kants. Meta: Research in Hermeneutics, Phenomenology, and Practical Philosophy 8: 304-325.

De Palma, V. (2016b). Phänomenologie-Psychologie oder Ontologie? Bulletin d'analyse phénoménologique $12, \mathrm{~N}^{\circ} 8: 1-28$.

De Palma, V. (2021). Phenomenology and British empiricism. In De Santis, D., Hopkins, B., Majolino, C. (Eds.). The Routledge Handbook of Phenomenology and Phenomenological Philosophy (73-86). New York: Routledge.

Holenstein, E. (1972). Phänomenologie der Assoziation. Zu Struktur und Funktion eines Grundprinzips der passiven Genesis bei E. Husserl. Den Haag: Nijhoff.

Husserl, E. (1972). Erfahrung und Urteil. Untersuchungen zur Genealogie der Logik. Hamburg: Meiner.

Kant, I. (1781/87). Kritik der reinen Vernunft. Riga: Hartknoch. 
Lask, E. (1923). Die Logik der Philosophie und die Kategorienlehre. In Gesammelte Schriften. Bd. II (1-282). Tübingen: Mohr.

Löwith, K. (1986). Vicos Grundsatz: verum et factum convertuntur. Seine theologische Prämisse und deren säkulare Konsequenzen. In Sämtliche Schriften. Bd. IX (195-227). Stuttgart: Metzler.

Maimon, S. (1790). Versuch über die Transzendentalphilosophie. Berlin: Voß.

Maimon, S. (1794). Die Kathegorien des Aristoteles. Berlin: Felisch.

Martin, G. (1969). Immanuel Kant. Ontologie und Wissenschaftstheorie. Berlin: De Gruyter.

Natorp, P. (1973). Zur Frage der logischen Methode. In Noack, H. (Hg.). Husserl (115). Darmstadt: Wissenschaftliche Buchgesellschaft.

Paton, H. J. (1936). Kant's Metaphysic of Experience. A Commentary on the First Half of the „, Kritik der reinen Vernunft“. Bd. I. London: Allen \& Unwin.

Puntel, L. B. (1983). Transzendentaler und absoluter Idealismus. In Henrich, D. (Hg.). Kant oder Hegel? Über Formen der Begründung in der Philosophie (198-229). Stuttgart: Klett-Cotta.

Scheler, M. (1966). Der Formalismus in der Ethik und die materiale Wertethik. In Gesammelte Werke. Bd. II. Bern/München: Franke.

Schelling, F. W. J. (1972). Grundlegung der positiven Philosophie. Münchner Vorlesung WS 1832/33 und SS 1833. Torino: Bottega d'Erasmo.

Sellars, W. (1981). Foundations for a Metaphysics of Pure Process. The Monist 64: 3 90.

Stumpf, C. (1891). Psychologie und Erkenntnistheorie. Abhandlungen der Bayerischen Akademie der Wissenschaften, I Kl. XIX Bd. II Abt.: 465-508.

Stumpf, C. (1939/40). Erkenntnislehre. 2 Bde. Leipzig: Barth.

Tugendhat, E. (1967). Der Wahrheitsbegriff bei Husserl und Heidegger. Berlin: De Gruyter. 